\title{
Unsteady MHD Flow for Fractional Casson Channel Fluid in a Porous Medium: An Application of the Caputo-Fabrizio Time- Fractional Derivative
}

\author{
Pongsakorn Sunthrayuth $\mathbb{D}^{1},{ }^{1}$ A. A. Alderremy, ${ }^{2}$ Fazal Ghani, ${ }^{3}$ Ayékotan M. J. Tchalla $\mathbb{D},{ }^{4}$ \\ Shaban Aly, ${ }^{5}$ and Yasser Elmasry ${ }^{6}$ \\ ${ }^{1}$ Department of Mathematics and Computer Science, Faculty of Science and Technology, Rajamangala University of Technology \\ Thanyaburi (RMUTT), Thanyaburi, Pathum Thani, Thailand \\ ${ }^{2}$ Department of Mathematics, Faculty of Science, King Khalid University, Abha 61413, Saudi Arabia \\ ${ }^{3}$ Department of Mathematics, Abdul Wali Khan University Mardan, Pakistan \\ ${ }^{4}$ Département de Mathématiques, Faculté des Sciences, Université de Lomé, 01 BP 1515, Lomé, Togo \\ ${ }^{5}$ Department of Mathematics, Faculty of Science, Al-Azhar University, Assiut, Egypt \\ ${ }^{6}$ Department of Mathematics, Faculty of Science, Mansoura University, Egypt
}

Correspondence should be addressed to Ayékotan M. J. Tchalla; atchalla@univ-lome.tg

Received 13 July 2021; Revised 25 August 2021; Accepted 20 January 2022; Published 2 March 2022

Academic Editor: Nehad Ali Shah

Copyright (C) 2022 Pongsakorn Sunthrayuth et al. This is an open access article distributed under the Creative Commons Attribution License, which permits unrestricted use, distribution, and reproduction in any medium, provided the original work is properly cited.

\begin{abstract}
Theoretically, this work describes the exact solutions of fractional Casson fluid through a channel under the effect of MHD and porous medium. The unsteady fluid motion of the bottom plate, which is confined by parallel but perpendicular sidewalls, supports the flow. By introducing the dimensionless parameters and variables, the momentum equation, as well as the initial and boundary conditions, has been transformed to a dimensionless form. A mix of Laplace and Fourier transformations is used to get the exact solution for the momentum equation. The constitutive equations for Caputo-Fabrizio's time-fractional derivative are also incorporated for recovering the exact solutions of the flow problem under consideration. After recovering the exact solutions for flow characteristics, three different cases at the surface of the bottom plate are discussed, by addressing the limiting cases under the influence of the side walls. Moreover, these solutions are captured graphically, and the effects of the Reynolds number Re, fractional parameter $\alpha$, effective permeability $K_{\text {eff }}$, and dimensionless parameter for Casson fluid $\beta$ on the fluid's motion are observed.
\end{abstract}

\section{Introduction}

Fractional calculus plays a critical part in the solving of complicated engineering issues. Because of its significance, a fractional model solution for flow issues is preferred by many scientists and researchers. A fraction model correctly depicts the motion of a flow issue when compared to ordinary differential equations (ODEs). It recovers an ODE's solution, which explains minute flow system fluctuations. Even for Newtonian fluids, the older scientists' operators including the Caputo operator employed a solitary kernel that resulted in complicated series solutions. In 2015,
Caputo and Fabrizio [1] suggested a new fractional operator that may be employed in simple ways to solve this problem. Following that, a lot of scholars have applied this notion to numerous sorts of flows using varied geometries. Alshabanat et al. [2] proposed a new fractional derivative utilizing a nonsingular form kernel of exponential and trigonometric functions. Singh et al. [3] investigated a fractional epidemiological model for viral determination in the computer utilizing fractional derivatives and numerically solved the modeled issue using the iterative technique. Shah and Khan [4] have discussed heat transmission for an oscillating second-grade fluid upon a vertical surface by employing 
the Caputo-Fabrizio derivative. In this work, the authors have determined the exact solutions for flow and thermal characteristics by applying Laplace transform. The authors of this investigation have also carried out a comparative study for the time derivative of fractional and integral order both for Newtonian and second-grade fluids and have highlighted that fractional parameter enhanced the flow characteristics due to augmented velocities of fractional fluid.

Researchers have been studying mathematical models for non-Newtonian fluids because of the growing trend in technological and industrial applications. Because of their relevance at the industrial level, research into these fluids is desirable. Non-Newtonian fluids, for example, lubricant production for a variety of vehicles, spinning of metal, metal extrusion, removing nonmetallic inclusions from molten metal, food, shoe manufacturing (the shoe must be filled with a nonNewtonian fluid to protect the feet from damage), and industries that deal with medicine and coolant, have a wide range of uses. Casson fluid is known as Non-Newtonian fluid because of its rheological features. Casson's model, introduced in 1959, was shown to be extremely flexible and to best reflect the curves of silicon suspension, among other things [5]. Hussain et al. [6] used the shooting method to find the numerical solution of a Casson fluid by changing viscosity flows near a shrinking/extending sheet by slip effects in MHD stagnation point flow. Casson nanofluid flows hydromagnetically through a porous stretched cylinder under Newtonian heat and mass conditions which were discussed by Naqvi et al. [7]. Rao et al. [8] found the exact solution of Casson fluid near a plate which is infinite, exponentially accelerated, and vertical with the effect of MHD and porosity. An overview of numerical approaches for heat and mass transfer in Casson fluids is presented by Verma and Mondal [9]. Sheikh et al. [10] obtained exact solutions of free convection MHD flow of Casson fluid in a channel by using Laplace transform. A fractional model with the Mittag-Leffler memory for generalized Casson MHD fluid by Newtonian heating was discussed by Tassaddiq et al. [11]. Goud et al. [12] calculated the exact solution of natural convection MHD flow of Casson fluid near a perpendicular plate through a porous medium by finite element method. The behavior of a non-Newtonian micropolarCasson fluid pulsatile flow in a restricted channel influenced by Lorentz force according to Darcy's law is investigated by Ali et al. [13].

The flow in the channels is also more important. Because of its relevance, numerous academics have been drawn to explore channel flows during the previous few decades. Using the Dufour effect, Jha and Ajibade [14] examined heat and mass transfer for free convective fluid flow along a vertical channel. Free convection of transient flow on a flat surface was studied by Ingham [15]. The authors of this study focused on a vertically oriented flat plate. Free convective flow with MHD effect on a flat plate was examined by Raptis and Singh [16]. The authors employed an accelerated vertical plate in this investigation. Fluid flow near an exponential plate has been explored by Singh and Kumar [17]. MHD fluid flow across a flat plate was studied by Khan et al. [18]. The authors of this study looked at the effect of sidewalls on fluid flow. Haq et al. [19] explored the flow of
MHD fluid on a porous sheet. Fetecau [20] has solved the fluid flow via a pipe analytically. The author used the Steklov expansion theorem to find the exact answer in this study. Furthermore, the same flow has been explored under the impact of side walls in this work by addressing the limiting instances. Using the Fourier transform, Fetecău and Zierep [21] examined a set of accurate solutions for the secondgrade fluid modeled issue. An abrupt jerk was applied to the fluid in this experiment to get fluid motion.

This work investigates a time-dependent fractional Casson fluid through a channel with porosity and MHD effect; the flow is caused by the bottom plate's unstable motion, which is confined by sidewalls that are parallel to one another but normal to the bottom plate. For the aim of creating a dimensionless form of governing equations, a group of nondimensional variables has been used in the momentum equation and applied boundary conditions. The exact solutions for the momentum equation were then obtained using integral transforms [22, 23] such as Laplace, finite Fourier, and Fourier transforms, as well as the Caputo-Fabrizio fractional derivative. These ideas were then addressed for various bottom plate instances. We utilized Mathcad 15 to explain the graphical results for the modeled issue after recovering the exact answers for various scenarios. The impact of different parameters involved in the solution of the flow problem has been discussed upon flow characteristics.

\section{Physical Description of Problem}

Assume that a fractional unsteady Casson fluid flows on an infinite plate. The plate is limited by two sidewalls that are parallel to each other but normal to the bottom plate and are separated by a distance of $d$. The flow is caused by the bottom plate because when $t=0$, both the plate and the fluid are at rest, and the bottom plate begins to move at an unsteady velocity $U_{0} f(t)$, which satisfies the condition $f(t)$ $=0$ at $t=0$ and piecewise continuous and exponential order at infinity. Because the motion is unidirectional, the equation describes the velocity of the flow system $V=u(y, z, t) i$ such that $v=w=0$.

Assumed flow system's governing equations [24] are as follows:

$\rho \frac{\partial u}{\partial t}=\left(1+\frac{1}{\xi}\right)\left(\frac{\partial \tau_{x y}}{\partial y}+\frac{\partial \tau_{x z}}{\partial z}\right)-\left(\sigma B_{o}^{2}+\frac{\mu \varphi}{k}\right) u, \quad y>0, \quad 0 \leq z \leq d$,

where

$$
\begin{aligned}
& \tau_{x y}=\mu \frac{\partial u}{\partial y} \\
& \tau_{x z}=\mu \frac{\partial u}{\partial z}
\end{aligned}
$$

The initial and boundary conditions for the problem are as follows: 


$$
\begin{aligned}
\tau_{x y}(y, z, 0) & =\tau_{x z}(y, z, 0)=0 \\
u(y, z, 0) & =0 \\
u(0, z, t) & =U_{0} f(t) \\
u(y, 0, t) & =u(y, d, t)=0
\end{aligned}
$$

although

$$
u(y, z, t) \longrightarrow 0, \frac{\partial u(y, z, t)}{\partial y} \longrightarrow 0, \quad \text { for } \quad y \longrightarrow \infty
$$

where $\mu$ represents the dynamic viscosity and $\rho$ is the density. Also, $f(t)=0$ at $t=0$ and $f(t) \neq 0$ for $t>0$.

Consider the collection of dimensionless variables to transform the governing equation and its initial boundary conditions for the postulated flow problem into nondimensional form.

$$
\begin{aligned}
y^{*} & =\frac{y}{d_{0}}, \\
z^{*} & =\frac{z}{d_{0}}, \\
\left(\tau_{x y}^{*}, \tau_{x z}^{*}\right) & =\frac{\left(\tau_{x y}, \tau_{x z}\right)}{\rho U_{0}^{2}}, \\
u^{*} & =\frac{u}{U_{0}}, \\
t^{*} & =\frac{t U_{0}}{d_{0}}, \\
L^{*} & =\frac{d}{d_{0}} .
\end{aligned}
$$

By plugging Equation (5) into Equations (1) and (2), we get the following:

$$
\frac{\partial u}{\partial t}=\frac{1}{\beta}\left(\frac{\partial \tau_{x y}}{\partial y}+\frac{\partial \tau_{x z}}{\partial z}\right)-K_{\mathrm{eff}} u, \quad y>0, \quad 0 \leq z \leq L
$$

$$
\begin{aligned}
\tau_{x y} & =\frac{1}{\operatorname{Re}} \frac{\partial u}{\partial y} \\
\tau_{x z} & =\frac{1}{\operatorname{Re}} \frac{\partial u}{\partial z}
\end{aligned}
$$

Here, $K_{\text {eff }}=M+(1 / K)$ is the effective permeability; moreover, $1 / K=v \varphi d_{0} / k U_{0}$ is the porous medium inverse permeability, and $M=\sigma B_{0}^{2} d_{0} / \rho U_{0}$ is the magnetic parameter.
In a dimensionless form, the initial and boundary conditions are as follows:

$$
\begin{aligned}
u(y, z, t) & =0, \quad t=0, \\
u(y, z, t) & =f\left(\frac{d_{0} t}{U_{0}}\right)=f(t), \quad y=0, \\
u(y, 0, t) & =u(y, L, t)=0, \\
u(y, z, t) \longrightarrow 0 & \longrightarrow 0, \quad y \longrightarrow \infty
\end{aligned}
$$

The length characteristics $d_{0}$, Reynolds number $\mathrm{Re}=$ $U_{0} d_{0} / v$, and dimensionless Casson fluid parameters are depicted above.

In a generalized form, the fractional constitutive equations are written as follows:

$$
\begin{aligned}
\tau_{x y} & =\frac{1}{\operatorname{Re}}{ }^{C F} D_{t}^{\alpha} \frac{\partial u}{\partial y}, \\
\tau_{x z} & =\frac{1}{\operatorname{Re}}{ }^{C F} D_{t}^{\alpha} \frac{\partial u}{\partial z} .
\end{aligned}
$$

\section{Problem Solution}

Using the Laplace transform to solve Equations (6) and (7) and incorporating Equation (9), we have the following:

$$
\begin{aligned}
s \bar{u} & =\frac{1}{\beta}\left(\frac{\partial \bar{\tau}_{x y}}{\partial y}+\frac{\partial \bar{\tau}_{x z}}{\partial z}\right)-K_{\mathrm{eff}} \bar{u}, \\
\bar{\tau}_{x y} & =\frac{1}{\operatorname{Re}} \frac{s}{(1-\alpha) s+\alpha} \frac{\partial \bar{u}}{\partial y}, \\
\bar{\tau}_{x z} & =\frac{1}{\operatorname{Re}} \frac{s}{(1-\alpha) s+\alpha} \frac{\partial \bar{u}}{\partial z} .
\end{aligned}
$$

By simplifying the above equations, we have the following:

$$
\frac{\partial^{2} \bar{u}}{\partial y^{2}}+\frac{\partial^{2} \bar{u}}{\partial z^{2}}=\frac{\operatorname{Re} \beta\left(s+K_{\mathrm{eff}}\right)((1-\alpha) s+\alpha)}{s} \bar{u}
$$

After using the Laplace transformation, the initial and boundary conditions were reduced to

$$
\begin{aligned}
& \bar{u}(y, z, s)=0, \quad s=0, \\
& \bar{u}(y, z, s)=\bar{F}(s), \quad y=0, \\
& \bar{u}(y, z, s)=\bar{u}(y, z, s)=0, \quad z=0, \quad z=L, \\
& \bar{u}(y, z, s) \longrightarrow 0, \\
& \frac{\partial \bar{u}(y, z, s)}{\partial y} \longrightarrow 0, \quad y \longrightarrow \infty .
\end{aligned}
$$


After that, multiply Equation (11) by $\sqrt{2 / \pi} \int_{0}^{\infty} \int_{0}^{L} \sin ($ $\left.\psi_{n} z\right) \sin (y \chi) d z d y$ and using Equation (12), in which $\zeta_{n}=$ $n \pi / L$ and the resulting equation was rewritten as follows:

$$
\bar{u}_{s n}(\chi, s)=\sqrt{\frac{2}{\pi}}\left(\frac{1-(-1)^{n}}{\zeta_{n}}\right)\left[\frac{\chi s F(s)}{\operatorname{Re} \beta((1-\alpha) s+\alpha)\left(s+K_{\mathrm{eff}}\right)+s\left(\chi^{2}+\zeta_{n}^{2}\right)}\right] .
$$

Rewrite Equation (13) in the following form:

$$
\begin{aligned}
\bar{u}_{s n}(\chi, s)= & \sqrt{\frac{2}{\pi}}\left(\frac{1-(-1)^{n}}{\zeta_{n}}\right)\left[\frac{\chi F(s)}{\chi^{2}+\zeta_{n}^{2}}\right] \\
& -\sqrt{\frac{2}{\pi}}\left(\frac{1-(-1)^{n}}{\zeta_{n}}\right) \frac{\chi F(s)}{\chi^{2}+\zeta_{n}^{2}} \\
& \cdot\left[\frac{((1-\alpha) s+\alpha)\left(s+K_{\text {eff }}\right)}{((1-\alpha) s+\alpha)\left(s+K_{\text {eff }}\right)+\left(s\left(\chi^{2}+\zeta_{n}^{2}\right) / \operatorname{Re} \beta\right)}\right] .
\end{aligned}
$$

Making use of Laplace inverse transform along with convolution theorem, we have the following equation:

$$
\begin{aligned}
\bar{u}_{s n}(\chi, t)= & \sqrt{\frac{2}{\pi}}\left(\frac{1-(-1)^{n}}{\zeta_{n}}\right)\left[\frac{\chi f(t)}{\chi^{2}+\zeta_{n}^{2}}\right] \\
& +\sqrt{\frac{2}{\pi}\left(\frac{1-(-1)^{n}}{\zeta_{n}}\right)\left(f^{\prime \prime}(t)+F_{1} f^{\prime}(t)+F_{2} f(t)\right)} \\
& \cdot \frac{\chi}{2 A_{1 n}(\chi)\left(\chi^{2}+\zeta_{n}^{2}\right)} *\left(e^{-D_{1 n}(\chi)}-e^{-D_{2 n}(\chi)}\right) .
\end{aligned}
$$

In Equation (15), we have the following:

$$
\begin{aligned}
F_{1} & =\frac{(1-\alpha) K_{\mathrm{eff}}+\alpha}{(1-\alpha)}, \\
F_{2} & =\frac{\alpha K_{\mathrm{eff}}}{(1-\alpha)}, \\
D_{1 n}(\chi) & =\frac{C_{1 n}(\chi)}{2}+A_{1 n}(\chi), \\
D_{2 n}(\chi) & =\frac{C_{1 n}(\chi)}{2}-A_{1 n}(\chi), \\
A_{1 n}(\chi) & =\sqrt{\left(\frac{C_{1 n}^{2}(\chi)}{4}-E_{2}\right)}, \\
C_{1 n}(\chi) & =\frac{\operatorname{Re} \beta(1-\alpha) K_{\mathrm{eff}}+\operatorname{Re} \beta \alpha+\chi^{2}+\zeta_{n}^{2}}{\operatorname{Re} \beta(1-\alpha)} .
\end{aligned}
$$

After using Fourier inversion methods to solve Equation (15) and simplifying the resulting equation,

$$
\begin{aligned}
u(y, z, t)= & \frac{4 f(t)}{L} \sum_{n=1}^{\infty} \frac{e^{-\zeta_{m} y} \sin \left(\zeta_{m} z\right)}{\zeta_{m}} \\
& +\frac{4}{\pi L}\left(f^{\prime \prime}(t)+F_{1} f^{\prime}(t)+F_{2} f(t)\right) \sum_{n=1}^{\infty} \frac{\sin \left(\zeta_{m} z\right)}{\zeta_{m}} \\
& * \int_{0}^{\infty} \frac{\chi\left(e^{-D_{11 m}(\chi)}-e^{-D_{22 m}(\chi)}\right) \sin (y \xi)}{A_{11 m}(\chi)\left(\chi^{2}+\zeta_{m}^{2}\right)} d \chi .
\end{aligned}
$$

In Equation (17), $m=2 n-1$, while

$$
\begin{aligned}
& A_{11 m}(\chi)=\sqrt{\left(\frac{C_{11 m}^{2}(\chi)}{4}-F_{2}\right)}, \\
& C_{11 m}(\chi)=\frac{\operatorname{Re} \beta(1-\alpha) K_{\mathrm{eff}}+\operatorname{Re} \beta \alpha+\chi^{2}+\zeta_{m}^{2}}{\operatorname{Re} \beta(1-\alpha)}, \\
& D_{11 m}(\chi)=\frac{C_{11 m}(\chi)}{2}+A_{11 m}(\chi), \\
& D_{22 m}(\chi)=\frac{C_{11 m}(\chi)}{2}-A_{11 m}(\chi), \\
& A_{11 m}(\chi)=\sqrt{\left(\frac{C_{11 m}{ }^{2}(\chi)}{4}-F_{2}\right)} .
\end{aligned}
$$

Take $L=2 h$ by translating system of the coordinate axis by putting $z=z^{*}+h$ and $\xi_{m}=(2 n-1 / 2 h) \pi$ with $\xi_{m}=\zeta_{m}$ in Equation (17); after that, we have the following:

$$
\begin{aligned}
u(y, z, t)= & \frac{2 f(t)}{h} \sum_{n=1}^{\infty} \frac{e^{-\xi_{m} y}(-1)^{n+1} \cos \left(\xi_{m} z\right)}{\xi_{m}} \\
& +\frac{2}{\pi h}\left(f^{\prime \prime}(t)+F_{1} f^{\prime}(t)+F_{2} f(t)\right) \\
& \times \sum_{n=1}^{\infty} \frac{(-1)^{n+1} \cos \left(\xi_{m} z\right)}{\xi_{m}} \\
& * \int_{0}^{\infty} \frac{\chi\left(e^{-D_{1 m}(\chi)}-e^{-D_{2 m}(\chi)}\right) \sin (y \chi)}{A_{1 m}(\chi)\left(\chi^{2}+\xi_{m}^{2}\right)} d \chi,
\end{aligned}
$$

where

$$
\begin{aligned}
A_{1 m}(\chi) & =\sqrt{\left(\frac{C_{1 m}^{2}(\chi)}{4}-F_{2}\right)} \\
C_{1 m}(\chi) & =\frac{\operatorname{Re} \beta(1-\alpha) K_{\mathrm{eff}}+\operatorname{Re} \beta \alpha+\chi^{2}+\xi_{m}^{2}}{\operatorname{Re} \beta(1-\alpha)}, \\
D_{11 m}(\chi) & =\frac{C_{11 m}(\chi)}{2}+A_{11 m}(\chi) \\
D_{22 m}(\chi) & =\frac{C_{11 m}(\chi)}{2}-A_{11 m}(\chi)
\end{aligned}
$$




\section{Special Cases}

4.1. $h \longrightarrow \infty$ Means That Distance among the Sidewalls Will Be Maximized. When the distance between the sidewalls of a flow system is increased, the flow is no longer impacted by these sidewalls and Equation (19) is reduced to the following:

$$
\begin{aligned}
u(y, t)= & f(t)+\frac{1}{\pi}\left(f^{\prime \prime}(t)+F_{1} f^{\prime}(t)+F_{2} f(t)\right) \\
& * \int_{0}^{\infty} \frac{\left(e^{-L_{1}(\chi) t}-e^{-L_{2}(\chi) t}\right) \sin (y \chi)}{\chi c_{1}(\chi)} d \chi .
\end{aligned}
$$

In Equation (21),

$$
\begin{aligned}
& A_{2}(\chi)=\sqrt{\left(\frac{C_{2}^{2}(\chi)}{4}-F_{2}\right)} \\
& C_{2}(\chi)=\frac{\operatorname{Re} \beta(1-\alpha) K_{\mathrm{eff}}+\operatorname{Re} \beta \alpha+\chi^{2}}{\operatorname{Re} \beta(1-\alpha)}, \\
& L_{1}(\chi)=\frac{C_{2}(\chi)}{2}+A_{2}(\chi) \\
& L_{2}(\chi)=\frac{C_{2}(\chi)}{2}-A_{2}(\chi) .
\end{aligned}
$$

4.2. Stokes' First Problem When $f(t)=H(t)$. By choosing $f$ $(t)=H(t)$ in Equation (19) (where $H(t)$ is Heaviside's unit step function), we will get the following:

$$
\begin{aligned}
u(y, z, t)= & \frac{2}{h} H(t) \sum \frac{(-1)^{n+1} e^{-\xi_{m} y} \cos \left(\xi_{m} z\right)}{\xi_{m}}+\frac{2}{h \pi} \sum_{n=1}^{\infty} \frac{(-1)^{n+1} \cos \left(\xi_{m} z\right)}{\xi_{m}} \\
& \times \int_{0}^{\infty} \frac{\chi\left[D_{2 m}(\chi)\left(D_{1 m}(\chi) F_{1}-D_{1 m}^{2}(\chi)-F_{2}\right) e^{-D_{1 m}(\chi) t}-D_{1 m}(\chi)\left(D_{2 m}(\chi) F_{1}-D_{2 m}^{2}(\chi)-F_{2}\right) e^{-D_{2 m}(\chi) t}\right] \sin (y \chi)}{D_{1 m}(\chi) D_{2 m}(\chi) A_{1 m}(\chi)\left(\chi^{2}+\xi_{m}^{2}\right)} d \chi
\end{aligned}
$$

If the side walls are ignored, Equation (23) becomes the following:

$u(y, t)=H(t)+\frac{1}{\pi} \int_{0}^{\infty} \frac{\left(\begin{array}{c}L_{2}(\chi)\left(L_{1}(\chi) F_{1}-L^{2}{ }_{1}(\chi)-F_{2}\right) e^{-L_{1}(\chi) t} \\ -L_{1}(\chi)\left(L_{2}(\chi) F_{1}-L_{2}^{2}(\chi)-F_{2}\right) e^{-L_{2}(\chi) t}\end{array}\right)}{\chi L_{1}(\chi) L_{2}(\chi) c_{1}(\chi)} \cdot \sin (y \chi) d \chi$.

4.3. If $f(t)=t^{a}$. By putting this case in Equation (19), after simplification, we will obtain the following:

$$
u(y, z, t)=\frac{2}{h} t^{a} \sum_{n=1}^{\infty} \frac{(-1)^{n+1} e^{-\xi_{m} y} \cos \left(\xi_{m} z\right)}{\xi_{m}}
$$

$$
\begin{aligned}
& +\frac{2}{h \pi}\left(\frac{d^{2}\left(t^{a}\right)}{d t^{2}}+F_{1} \frac{d\left(t^{a}\right)}{d t}+F_{2} t^{a}\right) \\
& \times \sum_{n=1}^{\infty} \frac{(-1)^{n+1} \cos \left(\xi_{m} z\right)}{\xi_{m}} \\
& * \int_{0}^{\infty} \frac{\chi\left(e^{-D_{1 m}(\chi) t}-e^{-D_{2 m}(\chi) t}\right) \sin (y \chi)}{c_{1 m}(\chi)\left(\chi^{2}+\xi_{m}{ }^{2}\right)} d \chi .
\end{aligned}
$$

The solution for an accelerating fractional Casson fluid is given by Equation (25). If the bottom plate is constantly accelerated, then $f(t)=t$; we get from Equation (19) that we obtain after simplification.

$$
\begin{aligned}
u(y, z, t)= & \frac{2}{h} t \sum_{n=1}^{\infty} \frac{(-1)^{n+1} e^{-\xi_{m} y} \cos \left(\xi_{m} z\right)}{\xi_{m}}+\frac{2}{h \pi} \sum_{n=1}^{\infty} \frac{(-1)^{n+1} \cos \left(\xi_{m} z\right)}{\xi_{m}} \times \int_{0}^{\infty} \frac{\chi \sin (y \chi)}{c_{1 m}(\chi)\left(\chi^{2}+\xi_{m}{ }^{2}\right)} \\
& \cdot\left[\begin{array}{c}
\frac{F_{1}}{D_{1 m}(\chi) D_{2 m}(\chi)}\left(D_{1 m}(\chi) e^{-D_{2 m}(\chi) t}-D_{2 m}(\chi) e^{-D_{1 m}(\chi) t}\right)+\frac{F_{2}}{D_{1 m}^{2}(\chi) D^{2}{ }_{2 m}(\chi)} \\
\left(-2 c_{1 m}(\chi)\left(D_{1 m}(\chi) D_{2 m}(\chi) t-A_{3 m}(\chi)\right)+D_{2 m}^{2}(\chi) e^{-D_{1 m}(\chi) t}-D_{1 m}^{2}(\chi) e^{-D_{2 m}(\chi) t}\right)
\end{array}\right] d \chi
\end{aligned}
$$



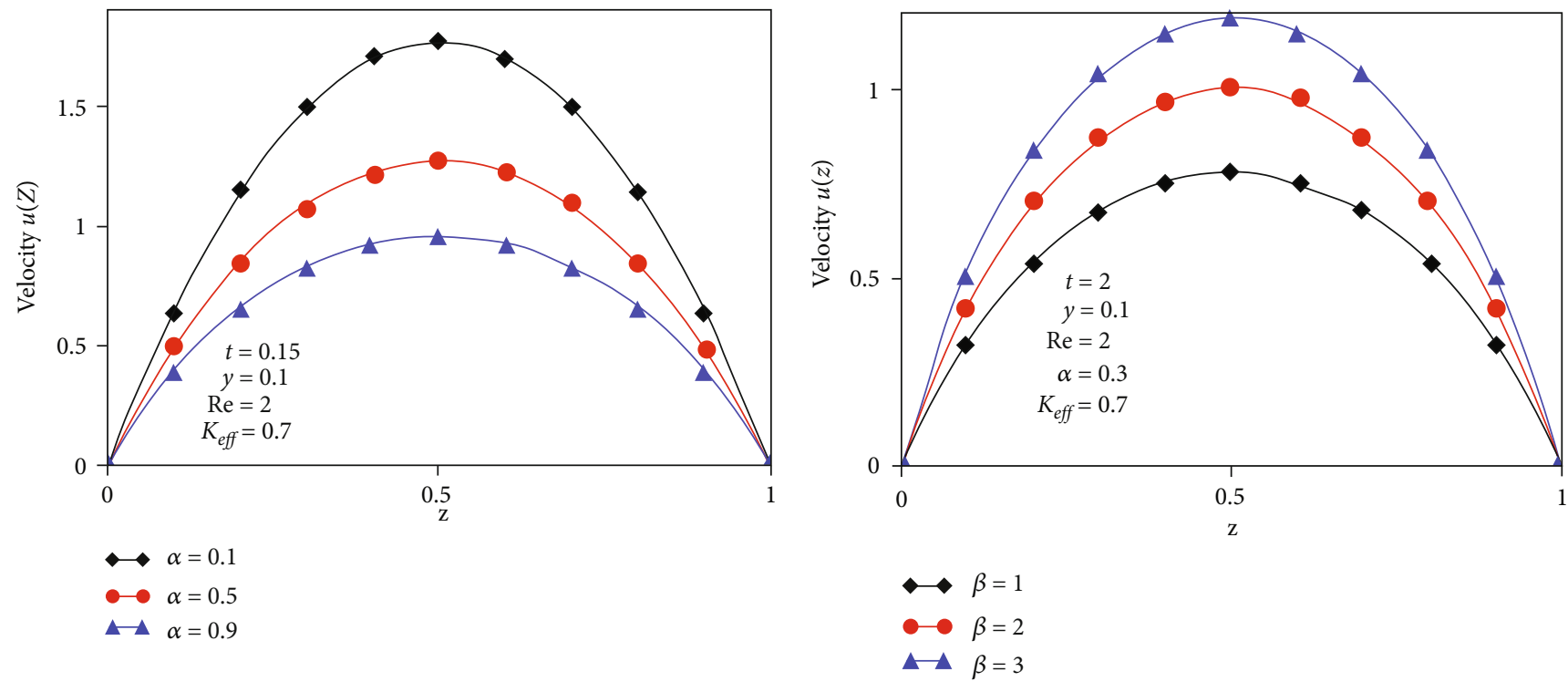

(a)
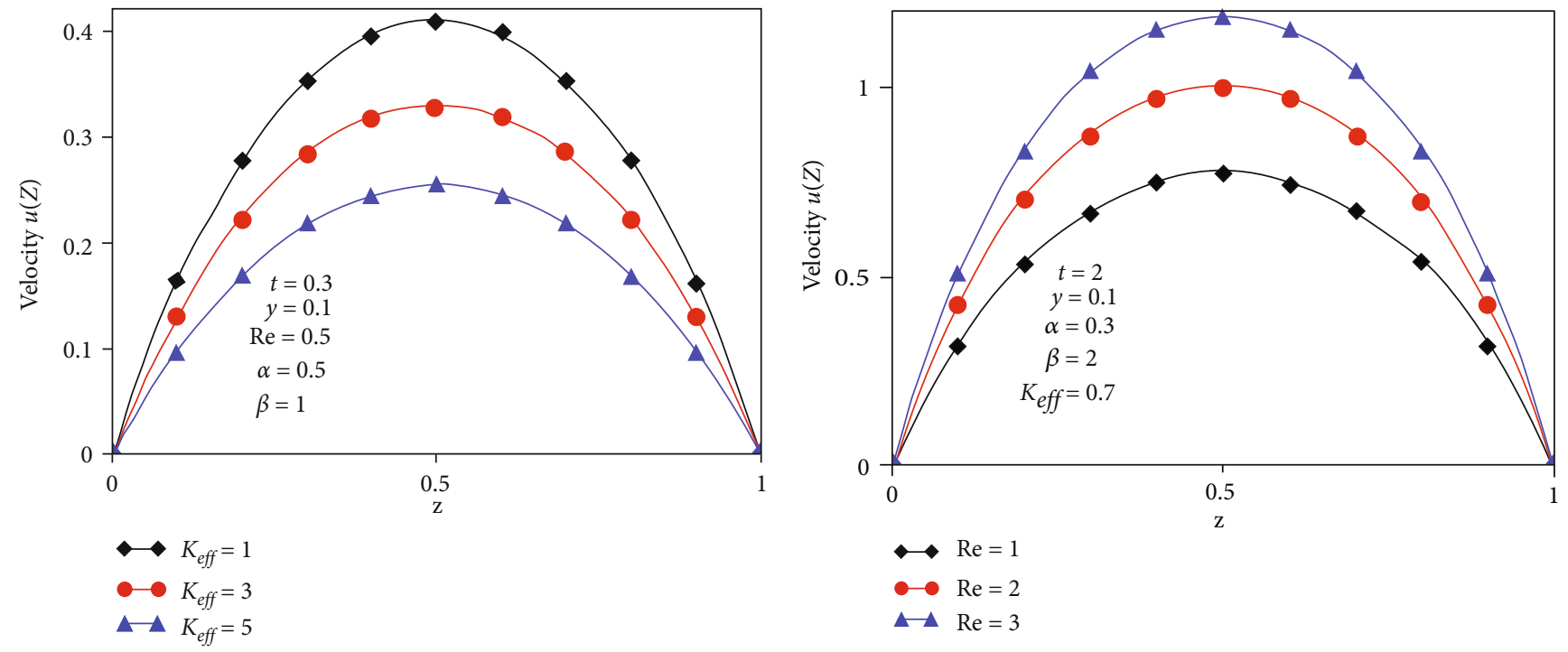

(c)

(d)

FIgURE 1: Flow characteristics for the impulsive type motion of fractional fluid when $z \in[0,1]$.

Again, by neglecting the effects of sidewalls that are $h$ $\longrightarrow \infty$ on fractional fluid flow, the velocity field takes the following form.

$$
\begin{aligned}
u(y, t)=t & +\frac{1}{\pi} \int_{0}^{\infty} \frac{\sin (y \chi)}{\chi c_{1}(\chi)} \\
& \cdot\left[\begin{array}{c}
\frac{F_{1}}{L_{1}(\chi) L_{2}(\chi)}\left(L_{1}(\chi) e^{-L_{2}(\chi) t}-L_{2}(\chi) e^{-L_{1}(\chi) t}\right)+\frac{F_{2}}{L^{2}{ }_{1}(\chi) L^{2}{ }_{2}(\chi)} \\
\left(-2 c_{1}(\chi)\left(L_{1}(\chi) L_{2}(\chi) t-A_{3}(\chi)\right)+L^{2}{ }_{2}(\chi) e^{-L_{1}(\chi) t}-L^{2}{ }_{1}(\chi) e^{-L_{2}(\chi) t}\right)
\end{array}\right] d \xi .
\end{aligned}
$$

4.4. $f(t)=H(t) e^{i \omega t}$. After the oscillation of the bottom plate, the fractional Casson fluid has oscillated with veloc- ities $u_{s}(y, z, t)$ and $u_{c}(y, z, t)$, respectively, corresponding to sine and cosine oscillations. This case gives the following:

$$
\begin{aligned}
u_{c s}(y, z, t)= & \frac{2}{h} \cos (\omega t) \sum_{n=1}^{\infty} \frac{(-1)^{n+1} e^{-\xi_{m} y} \cos \left(\xi_{m} z\right)}{\xi_{m}} \\
& +\frac{2}{h \pi} \sum_{n=1}^{\infty} \frac{(-1)^{n+1} \cos \left(\xi_{m} z\right)}{\xi_{m}} \int_{0}^{\infty} \frac{\chi \sin (y \chi)}{A_{1 m}(\chi)\left(\chi^{2}+\xi_{m}^{2}\right)} \\
& \times\left(\begin{array}{l}
\left\{\left(D_{1 m}(\chi) D_{2 m}(\chi)-\omega^{2}\right)\left(\omega^{2}-F_{2}\right)-F_{1} \omega^{2} C_{1 m}(\chi)\right\} \cos (\omega t) \\
+\left\{C_{1 m}(\chi)\left(\omega^{2}-F_{2}\right)+F_{1} D_{1 m}(\chi) D_{2 m}(\chi)-F_{1} \omega^{2}\right\} \sin (\omega t)
\end{array}\right) \\
& \times \frac{-2 A_{1 m}(\chi) d \chi}{\left(D_{1 m}^{2}(\chi)+\omega^{2}\right)\left(D_{2 m}^{2}(\chi)+\omega^{2}\right)},
\end{aligned}
$$




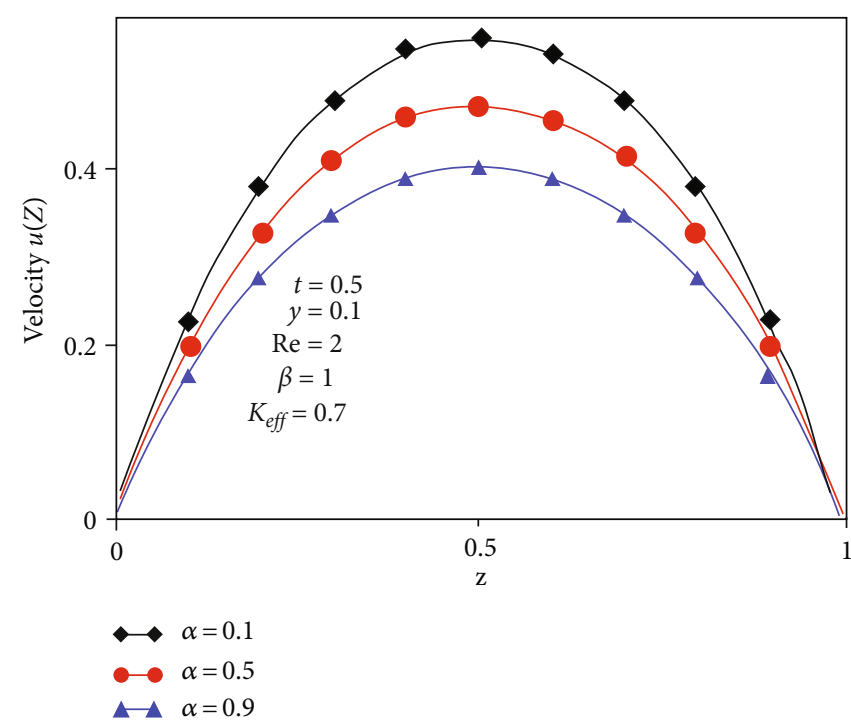

(a)

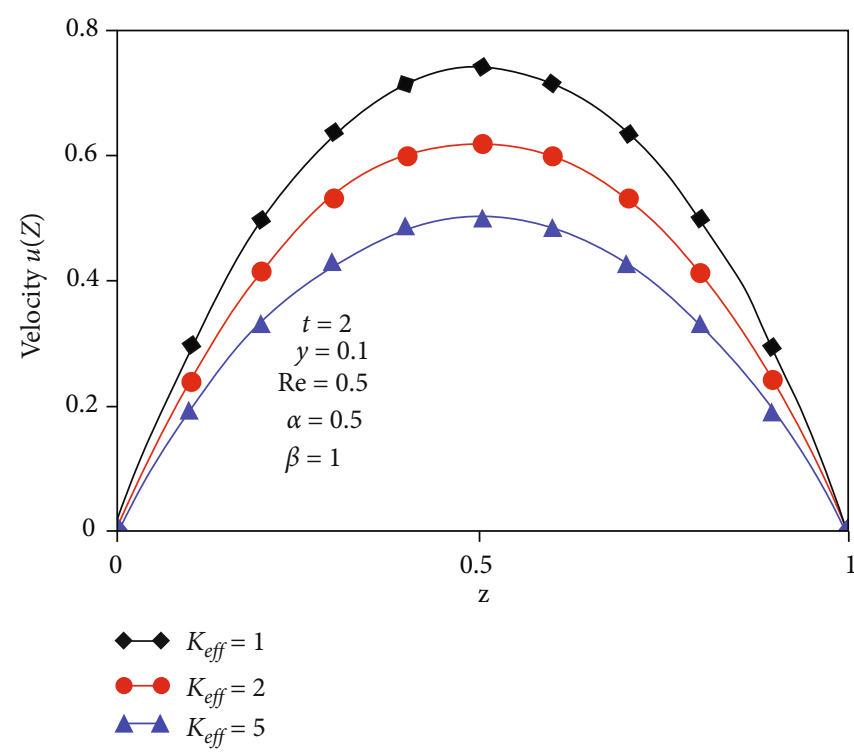

(c)

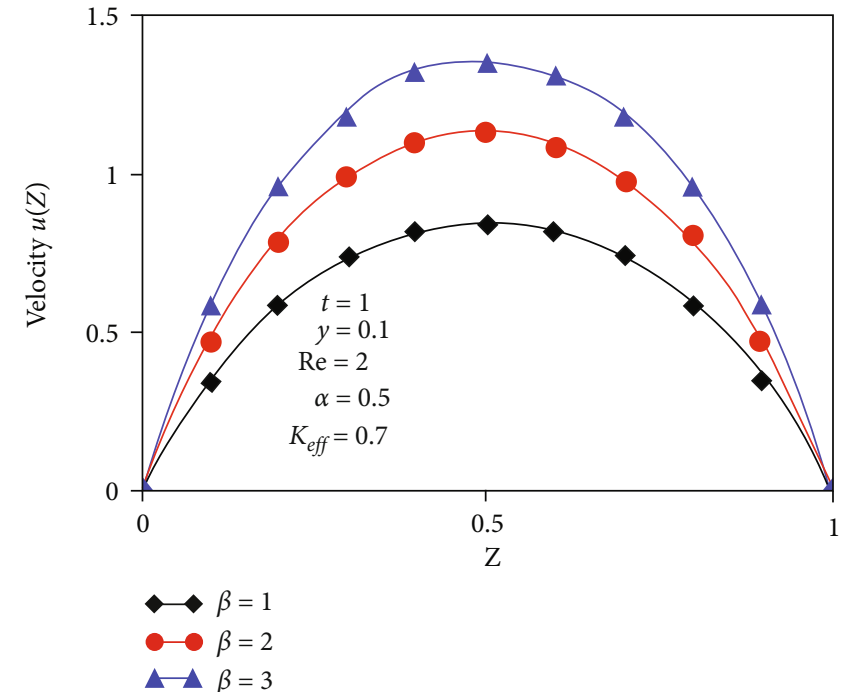

(b)

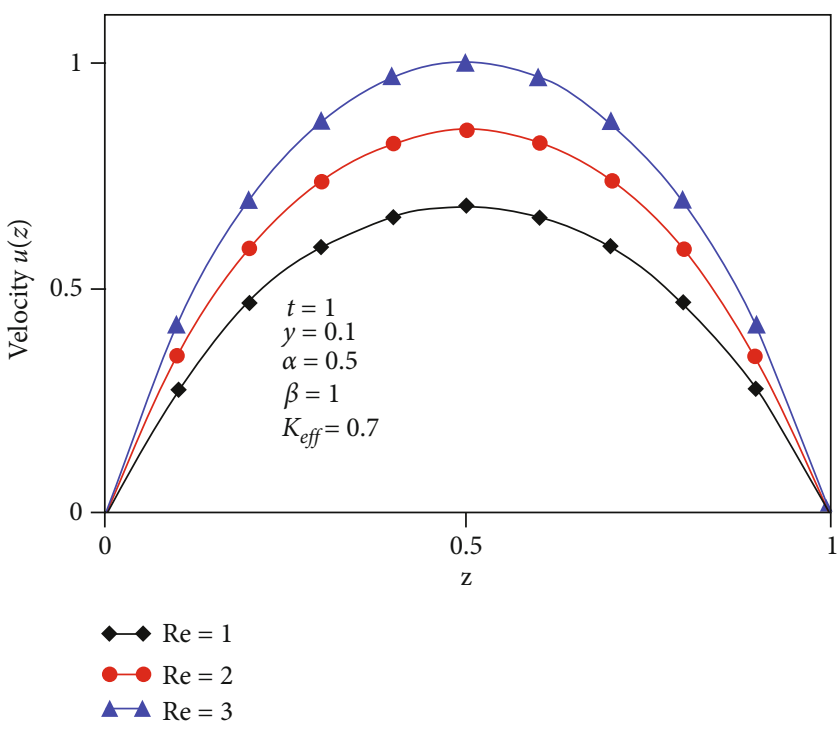

(d)

FIgURE 2: Flow characteristics for constant acceleration of fractional fluid when $z \in[0,1]$.

$$
\begin{aligned}
u_{c t}(y, z, t)= & \frac{2}{h \pi} \sum_{n=1}^{\infty} \frac{(-1)^{n+1} \cos \left(\xi_{m} z\right)}{\xi_{m}} \int_{0}^{\infty} \frac{\chi \sin (y \chi)}{A_{1 m}(\chi)\left(\chi^{2}+\xi_{m}^{2}\right)} \\
& \times\left[\frac{D_{1 m}(\chi)\left(F_{2}-\omega^{2}\right)+F_{1} \omega^{2}}{D_{1 m}^{2}(\chi)+\omega^{2}} e^{-D_{1 m}(\chi) t}\right. \\
& \left.+\frac{D_{2 m}(\chi)\left(F_{2}-\omega^{2}\right)-F_{1} \omega^{2}}{D_{2 m}^{2}(\chi)+\omega^{2}} e^{-D_{2 m}(\chi) t}\right] d \chi \\
u_{s s}(y, z, t)= & \frac{2}{h} \sin (\omega t) \sum_{n=1}^{\infty} \frac{(-1)^{n+1} e^{-\xi_{m} y} \cos \left(\xi_{m} z\right)}{\xi_{m}} \\
& +\frac{2}{h \pi} \sum_{n=1}^{\infty} \frac{(-1)^{n+1} \cos \left(\xi_{m} z\right)}{\xi_{m}} \int_{0}^{\infty} \frac{\chi \sin (y \chi)}{A_{1 m}(\chi)\left(\chi^{2}+\xi_{m}^{2}\right)}
\end{aligned}
$$

$$
\begin{gathered}
\times\left(\begin{array}{c}
\left\{\left(D_{1 m}(\chi) D_{2 m}(\chi)-\omega^{2}\right)\left(\omega^{2}-F_{2}\right)-F_{1} \omega^{2} C_{1 m}(\chi)\right\} \sin (\omega t) \\
+\left\{F_{1} \omega\left(\omega^{2}-D_{1 m}(\chi) D_{2 m}(\chi)\right)-\omega C_{1 m}(\chi)\left(\omega^{2}+F_{2}\right)\right\} \cos (\omega t)
\end{array}\right) \\
\times \frac{-2 A_{1 m}(\chi) d \chi}{\left(D_{1 m}^{2}(\chi)+\omega^{2}\right)\left(D_{2 m}^{2}(\chi)+\omega^{2}\right)}, \\
u_{s t}(y, z, t)=\frac{2}{h \pi} \sum_{n=1}^{\infty} \frac{(-1)^{n+1} \cos \left(\xi_{m} z\right)}{\xi_{m}} \int_{0}^{\infty} \frac{\chi \sin (y \chi)}{A_{1 m}(\chi)\left(\chi^{2}+\xi_{m}^{2}\right)} \\
\times\left[\frac{\omega\left(\omega^{2}+F_{1} D_{1 m}(\chi)-F_{2}\right)}{D_{1 m}^{2}(\chi)+\omega^{2}} e^{-D_{1 m}(\chi) t}\right. \\
\left.-\frac{\omega\left(\omega^{2}+F_{1} D_{2 m}(\chi)-F_{2}\right)}{D_{2 m}^{2}(\chi)+\omega^{2}} e^{-D_{2 m}(\chi) t}\right] d \chi .
\end{gathered}
$$




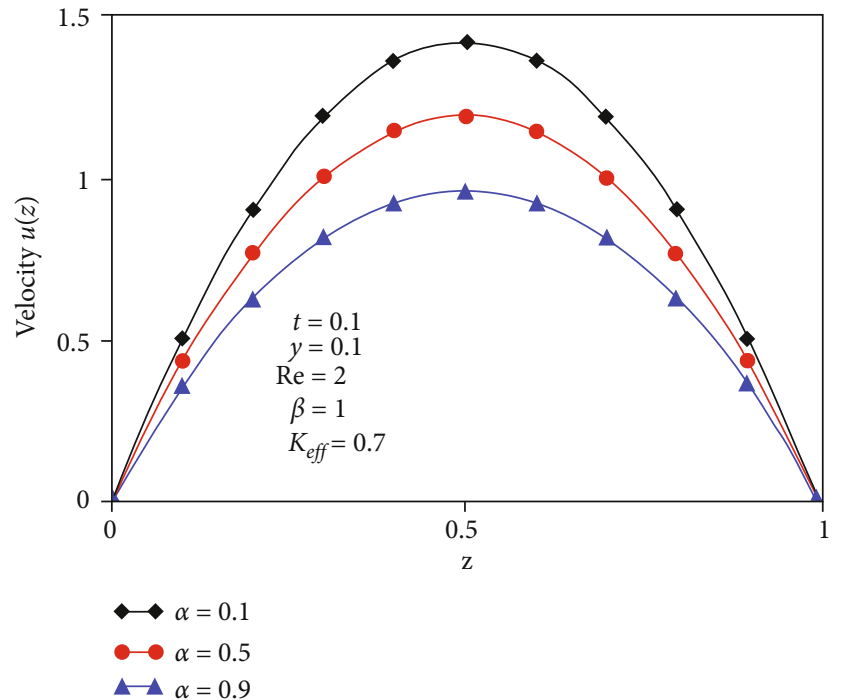

(a)

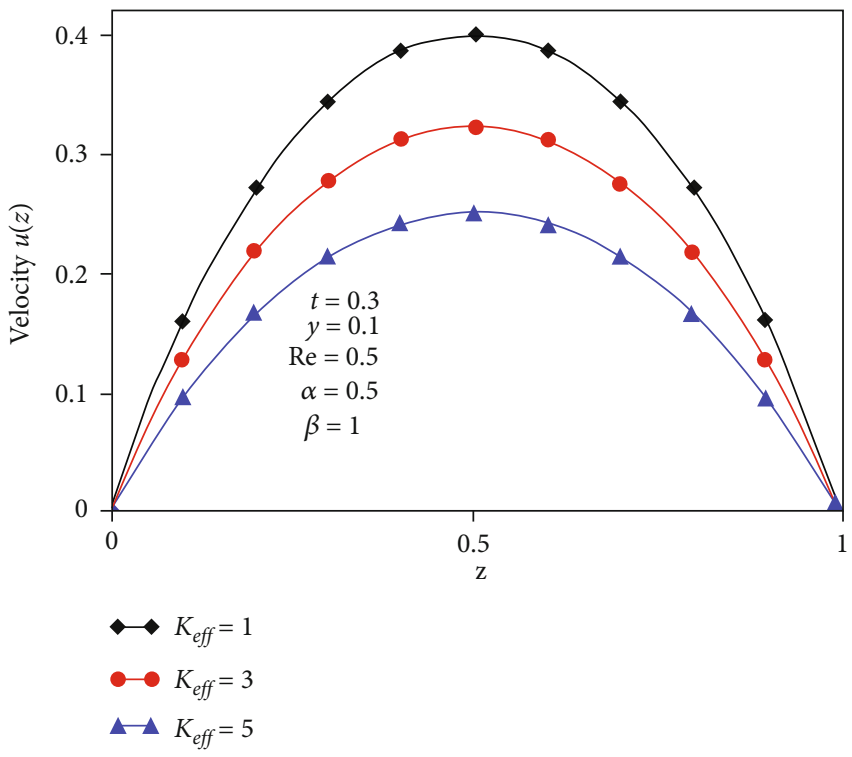

(c)

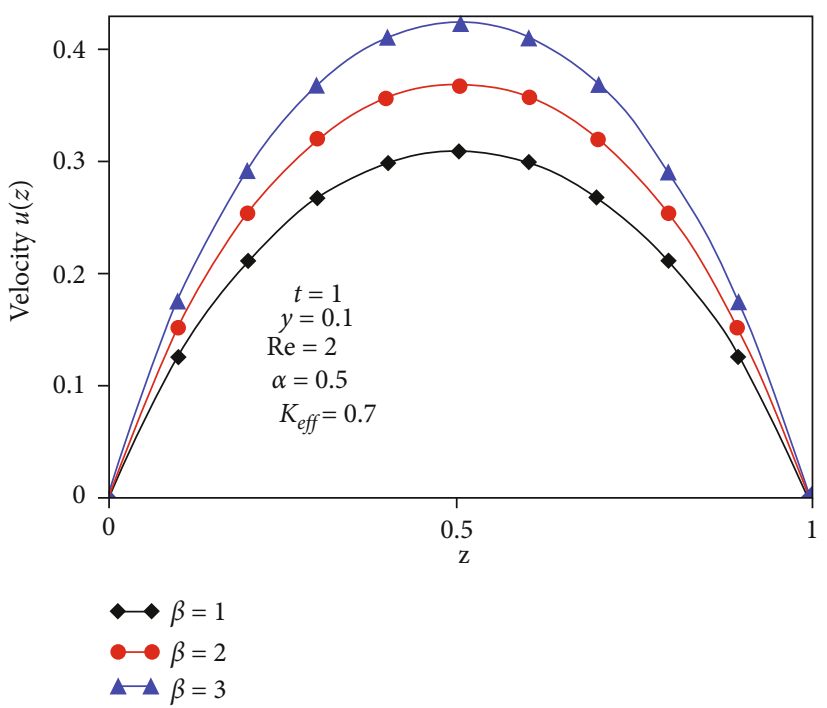

(b)

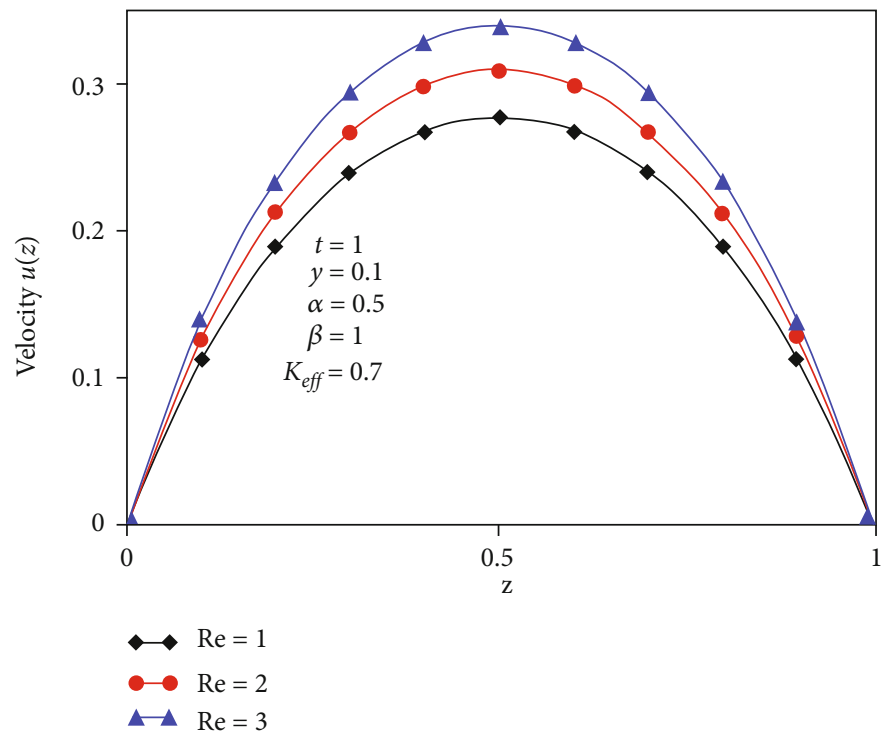

(d)

FIGURE 3: Flow characteristics for cosine oscillations of fractional fluid when $z \in[0,1]$.

Now, if we neglect the effects of sidewalls that are $h$ $\longrightarrow \infty$ on fractional fluid flow as a limiting case, then the velocity fields for such fluid flow caused by an oscillating bottom plate are as follows.

$$
\begin{aligned}
u_{c s}(y, z, t)= & \cos (\omega t)+\frac{1}{\pi} \int_{0}^{\infty} \frac{\sin (y \chi)}{\chi A_{2}(\chi)} \\
& \cdot\left(\begin{array}{l}
\left\{\left(L_{1}(\chi) L_{2}(\chi)-\omega^{2}\right)\left(\omega^{2}-F_{2}\right)-F_{1} \omega^{2} C_{2}(\chi)\right\} \cos (\omega t) \\
+\left\{C_{2}(\chi)\left(\omega^{2}-F_{2}\right)+F_{1} L_{1}(\chi) L_{2}(\chi)-F_{1} \omega^{2}\right\} \sin (\omega t)
\end{array}\right) \\
& \times \frac{-2 A_{2}(\chi) d \chi}{\left(L_{1}^{2}(\chi)+\omega^{2}\right)\left(L_{2}^{2}(\chi)+\omega^{2}\right)}, \\
u_{c t}(y, z, t)= & \frac{1}{\pi} \int_{0}^{\infty} \frac{\sin (y \chi)}{\chi A_{2}(\chi)}\left[\frac{L_{1}(\chi)\left(F_{2}-\omega^{2}\right)+F_{1} \omega^{2}}{L_{1}^{2}(\chi)+\omega^{2}} e^{-L_{1}(\chi) t}\right. \\
& \left.+\frac{L_{2}(\chi)\left(F_{2}-\omega^{2}\right)-F_{1} \omega^{2}}{L_{2}^{2}(\chi)+\omega^{2}} e^{-L_{2}(\chi) t}\right] d \chi
\end{aligned}
$$

$$
\begin{aligned}
u_{s s}(y, z, t)= & \sin (\omega \mathrm{t})+\frac{1}{\pi} \int_{0}^{\infty} \frac{\sin (y \chi)}{\chi A_{2}(\chi)} \\
& \cdot\left(\begin{array}{c}
\left\{\left(L_{1}(\chi) L_{2}(\chi)-\omega^{2}\right)\left(\omega^{2}-F_{2}\right)-E_{1} \omega^{2} C_{2}(\chi)\right\} \sin (\omega t) \\
+\left\{F_{1} \omega\left(\omega^{2}-L_{1}(\chi) L_{2}(\chi)\right)-\omega C_{2}(\chi)\left(\omega^{2}+F_{2}\right)\right\} \cos (\omega t)
\end{array}\right) \\
& \times \frac{-2 A_{2}(\chi) d \chi}{\left(L_{1}^{2}(\chi)+\omega^{2}\right)\left(L_{2}^{2}(\chi)+\omega^{2}\right)} \\
u_{s t}(y, z, t)= & \frac{1}{\pi} \int_{0}^{\infty} \frac{\sin (y \chi)}{\chi A_{2}(\chi)}\left[\frac{\omega\left(\omega^{2}+F_{1} L_{1}(\chi)-F_{2}\right)}{L_{1}^{2}(\chi)+\omega^{2}} e^{-L_{1}(\chi) t}\right. \\
& \left.-\frac{\omega\left(\omega^{2}+F_{1} L_{2}(\chi)-F_{2}\right)}{L_{2}^{2}(\chi)+\omega^{2}} e^{-L_{2}(\chi) t}\right] d \chi
\end{aligned}
$$

\section{Numerical Results and Discussions}

With the influence of MHD and porosity, three distinct forms of motion have been examined for fractional Casson fluid in a 


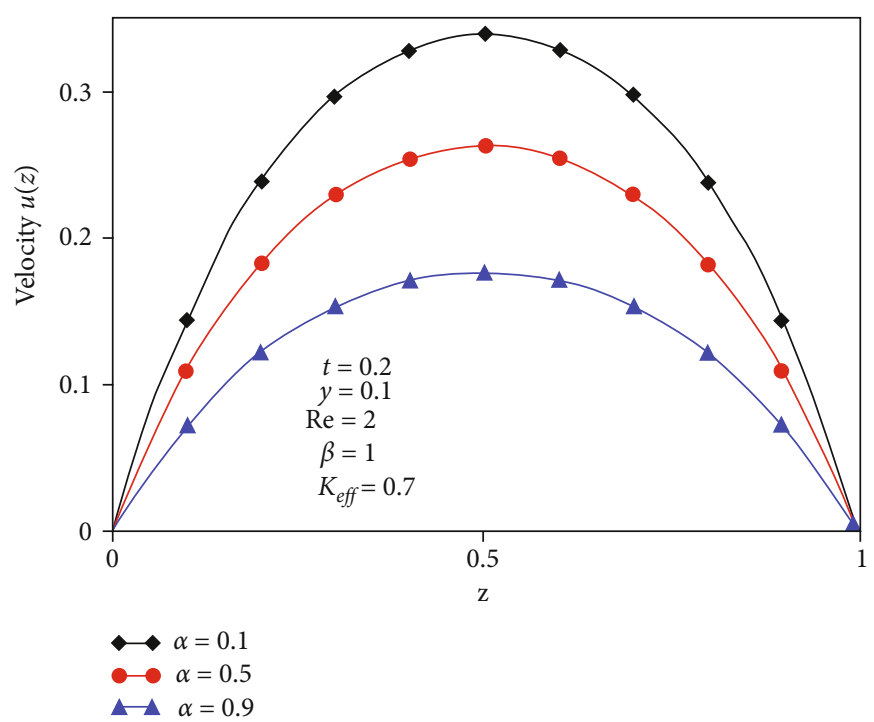

(a)

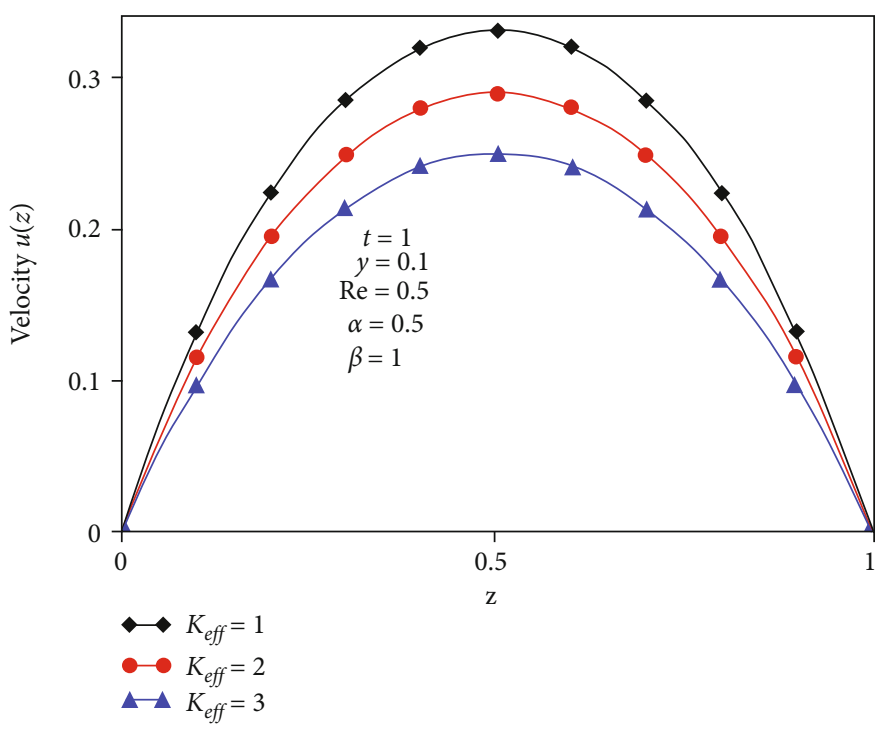

(c)

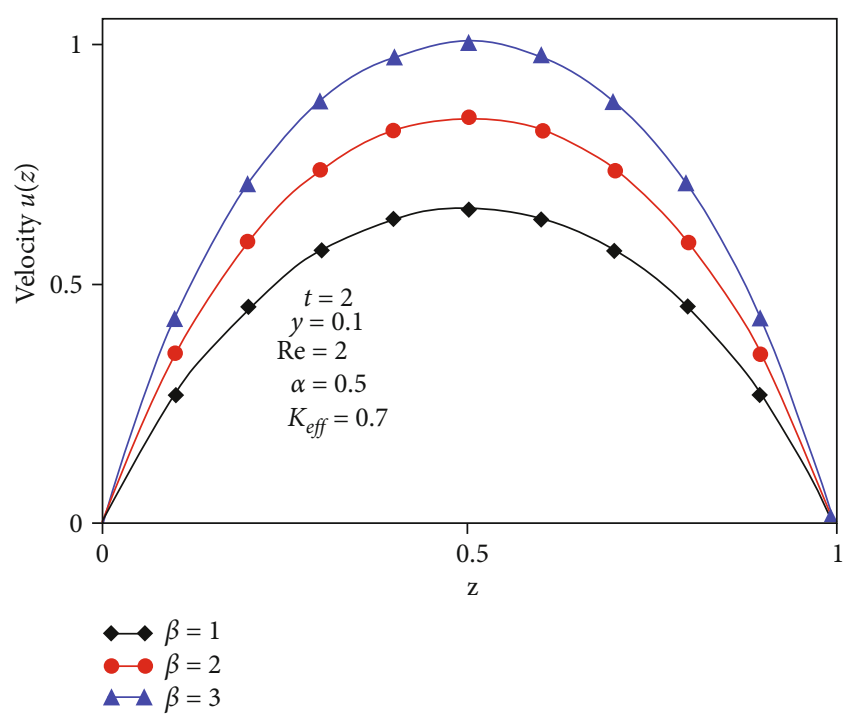

(b)

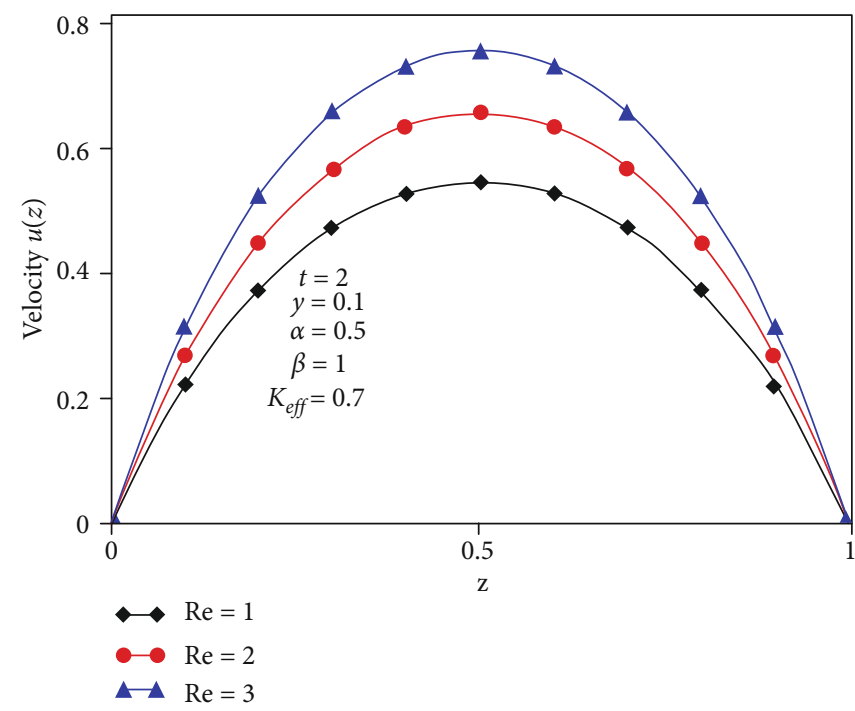

(d)

FIgURE 4: Flow characteristics for sine oscillations of fractional fluid when $z \in[0,1]$.

channel passing from a horizontal, unsteadily, and infinite moving plate that is confined by two parallel side walls separated by a distance $d$. For the objective of obtaining an exact solution, joint Laplace and Fourier transforms were used. For the problem under examination, many situations at the bottom plate have been studied. The same flow has been studied under the impact of side walls by addressing the limiting instances. After determining general solutions for any arbitrary flow, solution for impulsive, solution for constantly accelerated, and solution for the sinusoidal fluid motion of the bottom plate were retrieved.

Flow characteristics for Stokes' first issue are depicted in Figures 1(a)-1(d). It can be seen from these graphs that velocity decreases as the fractional parameter $(\alpha)$ increases because when the fractional parameter is increased, the fluid velocity decreases. An opposite impact is also observed for the dimensionless parameter for Casson fluid $(\beta)$ upon flow characteristics. It is also worth noting that velocity is a decreasing function of effective permeability $\left(K_{\text {eff }}\right)$, in these graphs. On the other hand, Reynolds number (Re) has the opposite effect on flow properties. The velocity of the fluid rises as the Reynolds number increases.

Figures 2(a)-2(d) show velocity profiles for fractional fluid flow with a ramping type. Figure 2(a) indicates that when $K_{\text {eff }}=0.7, \beta=1$, and $\operatorname{Re}=2$ then with decreasing values of fractional parameter $(\alpha)$, the characteristics of the flow are also improved. The velocity profile rises with increasing Casson fluid values, as seen in Figure 2(b). In Figure 2(c), the velocity decreases as the effective permeability increases. Similarly, when the Reynolds number $(\mathrm{Re})$ increases, the flow properties improve. As a result, the velocity profile is a decreasing function of the fractional and effective permeability parameters. 
The effects of cosine oscillations on flow characteristics are depicted in Figures 3(a)-3(d). In the instance of cosine oscillations, we can see that velocity decreases as the fractional parameter $(\alpha)$ and effective permeability $\left(K_{\text {eff }}\right)$ increase, and that flow profiles decrease as these two components increase which is observed from Figures 3(a) and 3(c). The flow profile is rising with greater values of the dimensionless parameter for Casson fluid parameter $(\beta)$ and Reynolds number (Re) as seen in Figures 3(b) and 3(d). The effect of sine oscillations on flow characteristics is seen in Figures 4(a)-4(d). For sine oscillations on flow characteristics, a similar effect has been seen for various values of significant parameters.

\section{Conclusions}

The precise solutions for a time-dependent fractional Casson fluid traversing a channel under MHD and porosity effects are described in this paper. The flow is caused by the bottom plate's unstable motion, which is restricted by parallel but perpendicular sidewalls. By using an appropriate set of dimensionless variables, the momentum equation, as well as the initial and boundary conditions, has been changed to a dimensionless form. A mix of Laplace and Fourier transformations is used to get the exact solution of the momentum equation. For the problem under examination, many situations at the bottom plate have been studied. The same flow has been studied under the impact of side walls by addressing the limiting instances. After determining general solutions for any arbitrary flow, solutions for impulsive, solutions for constant accelerated, and solutions for the sinusoidal fluid motion of the bottom plate were retrieved. These precise solutions are also visually depicted in support of our effort and then theoretically presented. The following points are emphasized after a thorough evaluation of the work.

(i) In this study, it was discovered that Reynolds number has a significant influence on flow characteristics since the velocity of the fluid increases as the Reynolds number grows in all limiting/special instances

(ii) Whether the bottom plate is at rest or moving by constant acceleration, the velocity of the fluid decreases as the fractional parameter and effective permeability increase for long periods of time

(iii) The velocity of cosine/sine oscillations increases with the rising Reynolds number and Casson parameter, since flow profiles increase with rising values of these two parameters, as seen in this work. The flow profile, on the other hand, decreases when the fractional parameter and effective permeability increase

\section{Data Availability}

The numerical data used to support the findings of this study are included within the article.

\section{Conflicts of Interest}

The authors declare that there are no conflicts of interest regarding the publication of this article.

\section{Acknowledgments}

The authors extend their appreciation to the Deanship of Scientific Research at King Khalid University, Saudi Arabia, for funding this work through research groups program under grant number (R.G.P1./278/42).

\section{References}

[1] M. Caputo and M. Fabrizio, "A new definition of fractional derivative without singular kernel," Progress in Fractional Differentiation and Applications, vol. 1, no. 2, pp. 1-13, 2015.

[2] A. Alshabanat, M. Jleli, S. Kumar, and B. Samet, "Generalization of Caputo-Fabrizio fractional derivative and applications to electrical circuits," Frontiers in Physics, vol. 8, p. 64, 2020.

[3] J. Singh, D. Kumar, Z. Hammouch, and A. Atangana, "A fractional epidemiological model for computer viruses pertaining to a new fractional derivative," Applied Mathematics and Computation, vol. 316, pp. 504-515, 2018.

[4] N. A. Shah and I. Khan, "Heat transfer analysis in a second grade fluid over and oscillating vertical plate using fractional Caputo-Fabrizio derivatives," The European Physical Journal C, vol. 76, no. 7, pp. 1-11, 2016.

[5] M. Wahiduzzaman, M. T. Islam, P. Sultana, and M. Afikuzzaman, "MHD Couette flow of a Casson fluid between parallel porous plates," Progress in Nonlinear Dynamics and Chaos, vol. 2, no. 2, pp. 51-60, 2014.

[6] A. Hussain, S. Afzal, R. Rizwana, and M. Y. Malik, "MHD stagnation point flow of a Casson fluid with variable viscosity flowing past an extending/shrinking sheet with slip effects," Physica A, vol. 553, p. 124080, 2020.

[7] S. M. R. S. Naqvi, T. Muhammad, and M. Asma, "Hydromagnetic flow of Casson nanofluid over a porous stretching cylinder with Newtonian heat and mass conditions," Physica A, vol. 550, p. 123988, 2020.

[8] S. R. Rao, G. Vidyasagar, and G. V. S. R. Deekshitulu, "Unsteady MHD free convection Casson fluid flow past an exponentially accelerated infinite vertical porous plate through porous medium in the presence of radiation absorption with heat generation/absorption," Materials Today: Proceedings, vol. 42, pp. 1608-1616, 2021.

[9] V. R. Verma and S. Mondal, "A brief review of numerical methods for heat and mass transfer of Casson fluids," Partial Differential Equations in Applied Mathematics, vol. 3, article 100034, 2021.

[10] N. A. Sheikh, D. L. C. Ching, I. Khan, D. Kumar, and K. S. Nisar, "A new model of fractional Casson fluid based on generalized Fick's and Fourier's laws together with heat and mass transfer," Alexandria Engineering Journal, vol. 59, no. 5, pp. 2865-2876, 2020.

[11] A. Tassaddiq, I. Khan, K. S. Nisar, and J. Singh, "MHD flow of a generalized Casson fluid with Newtonian heating: a fractional model with Mittag-Leffler memory," Alexandria Engineering Journal, vol. 59, no. 5, pp. 3049-3059, 2020.

[12] B. S. Goud, P. P. Kumar, and B. S. Malga, "Effect of heat source on an unsteady MHD free convection flow of Casson fluid past 
a vertical oscillating plate in porous medium using finite element analysis," Partial Differential Equations in Applied Mathematics, vol. 2, article 100015, 2020.

[13] A. Ali, M. Umar, Z. Bukhari, and Z. Abbas, "Pulsating flow of a micropolar-Casson fluid through a constricted channel influenced by a magnetic field and Darcian porous medium: a numerical study," Results Phys, vol. 19, p. 103544, 2020.

[14] B. K. Jha and A. O. Ajibade, "Free convection heat and mass transfer flow in a vertical channel with the Dufour effect," Journal of Mechanical Engineering, vol. 224, no. 2, pp. 91-101, 2010.

[15] D. B. Ingham, "Transient free convection on an isothermal vertical flat plate," International Journal of Heat and Mass Transfer, vol. 21, no. 1, pp. 67-69, 1978.

[16] A. Raptis and A. K. Singh, "MHD free convection flow past an accelerated vertical plate," International Communications in Heat and Mass, vol. 10, no. 4, pp. 313-321, 1983.

[17] A. K. Singh and N. Kumar, "Free-convection flow past an exponentially accelerated vertical plate," Astrophysics and Space Science, vol. 98, no. 2, pp. 245-248, 1984.

[18] M. Khan, R. Malik, and A. Anjum, "Analytic solutions for MHD flows of an Oldroyd-B fluid between two sidewalls perpendicular to the plate," Chemical Engineering Communications, vol. 198, no. 11, pp. 1415-1434, 2011.

[19] S. U. Haq, A. U. Rahman, I. Khan, F. Ali, and S. I. A. Shah, "The impact of side walls on the MHD flow of a secondgrade fluid through a porous medium," Neural Computing and Applications, vol. 30, no. 4, pp. 1103-1109, 2018.

[20] C. Fetecau, "Analytical solutions for non-Newtonian fluid flows in pipe-like domains," International Journal of NonLinear Mechanics, vol. 39, no. 2, pp. 225-231, 2004.

[21] C. Fetecău and J. Zierep, "On a class of exact solutions of the equations of motion of a second grade fluid," Acta Mechanica, vol. 150, no. 1-2, pp. 135-138, 2001.

[22] I. S. Gradshteyn and I. M. Ryzhik, Table of integrals, series, and products, Academic Press, Seventh edition, 2007.

[23] L. Debnath and D. Bhatta, Integral transforms and their applications, Chapman \& Hall/CRC Taylor \& Francis Group, Second edition, 2006.

[24] X. H. Zhang, R. Shah, S. Saleem, N. A. Shah, Z. A. Khan, and J. D. Chung, "Natural convection flow maxwell fluids with generalized thermal transport and newtonian heating," Case Studies in Thermal Engineering, vol. 27, p. 101226, 2021. 